\section{Huntington's disease is a four-repeat tauopathy with tau nuclear rods}

Marta Fernández-Nogales ${ }^{1,2}$, Jorge R Cabrera ${ }^{1,2,5}$, María Santos-Galindo 1,2,5, Jeroen J M Hoozemans ${ }^{3}$, Isidro Ferrer ${ }^{2,4}$, Annemieke J M Rozemuller ${ }^{3}$, Félix Hernández ${ }^{1,2}$, Jesús Avila ${ }^{1,2}$ \& José J Lucas ${ }^{1,2}$

An imbalance of tau isoforms containing either three or four microtubule-binding repeats causes frontotemporal dementia with parkinsonism linked to chromosome 17 (FTDP-17) in families with intronic mutations in the MAPT gene.

Here we report equivalent imbalances at the mRNA and protein levels and increased total tau levels in the brains of subjects with Huntington's disease (HD) together with rod-like tau deposits along neuronal nuclei. These tau nuclear rods show an ordered filamentous ultrastructure and can be found filling the neuronal nuclear indentations previously reported in HD brains. Finally, alterations in serine/arginine-rich splicing factor- 6 coincide with tau missplicing, and a role of tau in HD pathogenesis is evidenced by the attenuation of motor abnormalities of mutant HTT transgenic mice in tau knockout backgrounds.

Tauopathies are a group of neurodegenerative diseases, including Alzheimer's disease (AD), progressive supranuclear palsy, corticobasal degeneration, Pick's disease and FTDP-17, that are characterized by altered metabolism and deposition of the microtubule-associated protein tau ${ }^{1}$. Alternative splicing of exon 10 of the microtubule-associated protein tau $(M A P T)$ gene results in tau isoforms containing either three or four microtubule-binding repeats ( $3 \mathrm{R}$ and $4 \mathrm{R}$, respectively) ${ }^{2}$. Discovery of silent and intronic mutations leading to an increased $4 \mathrm{R} / 3 \mathrm{R}$ ratio in families affected by FTDP-17 (ref. 3) revealed that an imbalance in $4 \mathrm{R}$ and $3 \mathrm{R}$ in favor of the $4 \mathrm{R}$ isoform is sufficient to cause neurodegeneration with personality disturbances, dementia and motor dysfunction.

HD is an autosomal dominant neurodegenerative disorder characterized by involuntary movements, psychiatric symptoms and dementia that is caused by an expanded CAG repeat in exon 1 of the huntingtin (HTT) gene ${ }^{4}$. HD thus belongs to the group of dominant trinucleotide repeat diseases, which include many other CAG repeat disorders, such as spinocerebellar ataxia type 1 (SCA1), SCA2, SCA3, SCA6, SCA7, SCA12, SCA17, dentatorubropallidoluysian atrophy and spinobulbar muscular atrophy, as well as the CUG repeat disorders SCA8 and myotonic dystrophy type 1 (ref. 5). A key element in the pathogenesis of myotonic dystrophy type 1 is the binding of splicing factors by the mutant CUG transcript, thus leading to alternative splicing aberrations in multiple genes ${ }^{6}$. Notably, CAG repeats have recently been shown to mimic CUG repeats in the misregulation of alternative splicing ${ }^{7}$. As it has also been very recently reported that aberrant splicing contributes to the generation of the highly toxic short-N-terminal species of $\mathrm{HTT}^{8}$, it is conceivable that splicing alterations contribute substantially to HD pathogenesis.

Here we address whether the neurodegeneration-causing increase in $4 \mathrm{R} / 3 \mathrm{R}$ tau mRNA ratio occurs in HD. To test this, we performed quantitative RT-PCR from RNA extracted from striatum and cortex of subjects with HD and healthy control subjects. The number of tau mRNA copies per nanogram of total RNA was equivalent in control subjects and in subjects with HD in both striatum and cortex (Fig. 1a). However, in the striatum of control subjects, 3R tau mRNA isoforms predominated over $4 \mathrm{R}$ mRNA isoforms (59.1\% versus $40.9 \%$ ), whereas in HD samples, the proportion was shifted toward a higher content of $4 \mathrm{R}$ mRNA isoforms (46.3\% versus $53.7 \%$ ). Accordingly, the $4 \mathrm{R} / 3 \mathrm{R}$ isoform ratio was dramatically increased in $\mathrm{HD}$ samples compared to controls (1.77-fold increase, $P=0.002)$. In the cortex, $3 \mathrm{R}$ tau mRNA isoforms predominated over $4 \mathrm{R} \mathrm{mRNA}$ isoforms both in control and in HD samples. However, as the difference between $3 \mathrm{R}$ and $4 \mathrm{R}$ mRNA content was higher in control subjects $(62.8 \%$ versus $37.2 \%)$ than in subjects with $\mathrm{HD}$ (56.3\% versus $43.7 \%)$, the $4 \mathrm{R} / 3 \mathrm{R}$ ratio was also significantly increased in HD cortex (1.43-fold increase, $P=0.015$ ). To rule out any influence of the postmortem interval in this result, we confirmed that the $4 \mathrm{R} / 3 \mathrm{R}$ tau ratio stayed constant in RNAs from control and HD RNA samples that were stored for up to $48 \mathrm{~h}$ at $4{ }^{\circ} \mathrm{C}$ to force a decrease in RNA integrity (Supplementary Fig. 1).

We then used antibodies specific to the $3 \mathrm{R}$ and $4 \mathrm{R}$ protein isoforms (RD3 and RD4, respectively) to explore by western blotting whether the increase in the $4 \mathrm{R} / 3 \mathrm{R}$ mRNA ratio results in an equivalent imbalance in tau protein isoforms. HD samples showed a clear increase in $4 \mathrm{R}$ isoform levels in striatum and cortex and a clear decrease in $3 \mathrm{R}$ isoform levels, particularly in striatum (Fig. 1b). In good agreement with the mRNA data, the increase in $4 \mathrm{R} / 3 \mathrm{R}$ ratio was higher in striatum than in cortex (3.08-fold increase, $P=0.006$ and 2.1 -fold increase, $P=0.013$; respectively). Notably, whereas total tau content (as measured with Tau5 antibody) was similar in striatum of control subjects and subjects with HD, we observed a clear increase in total tau levels in the cortex of subjects with HD (1.52-fold increase, $P=0.016)$ with the appearance of additional low-molecular-weight ( 35 and $39 \mathrm{kDa}$ ) truncated forms of tau. We also observed this increase and the appearance of the 35 - and $39-\mathrm{kDa}$ bands with the $5 \mathrm{E} 2$ antibody that also recognizes total tau, thus confirming specificity of the low-molecular-weight bands (Supplementary Fig. 2).

${ }^{1}$ Center for Molecular Biology "Severo Ochoa” (CBMSO) Consejo Superior de Investigaciones Científicas y Universidad Autónoma de Madrid (CSIC/UAM), Madrid, Spain. ${ }^{2}$ Networking Research Center on Neurodegenerative Diseases (CIBERNED), Instituto de Salud Carlos III, Spain. ${ }^{3}$ Department of Pathology, Neuroscience Campus Amsterdam, VU University Medical Center, Amsterdam, the Netherlands. ${ }^{4}$ Institute of Neuropathology, Bellvitge Biomedical Research Institute (IDIBELL)University Hospital Bellvitge, University of Barcelona, Hospitalet de Llobregat, Barcelona, Spain. ${ }^{5}$ These authors contributed equally to the work. Correspondence should be addressed to J.J.L. (jjlucas@cbm.csic.es).

Received 11 December 2013; accepted 3 June 2014; published online 20 July 2014; doi:10.1038/nm.3617 
Figure 1 Increased 4R/3R tau isoform ratio and total tau protein content in HD brains, presence of TNRs in HD (and AD) brains and analysis of SRSF6 splicing factor in HD brains and in transfected human neuroblastoma SHSY5Y cells. (a) Quantitative RT-PCR analysis of total tau mRNA and of $3 R$ and $4 R$ mRNA isoforms in striatum and cortex of subjects with HD (striatum, $n=5$ for control and $n=9$ for HD; cortex, $n=9$ for control and $n=10$ for HD; Student's $t$-test; ${ }^{*} P<0.01,{ }^{*} P<0.05$; only samples with RNA integrity number (RIN) above 5 were included in the analysis).

(b) Western blot analysis of 3R and 4R tau isoforms with the RD3- and RD4-specific antibodies and of total tau with the Tau 5 antibody in total homogenates from striatum and cortex of control and HD brains (the position of the 49-kDa molecular weight marker is indicated; < signs indicate the truncated 35and 39-kDa bands; striatum, $n=13$ for control and $n=16$ for HD; cortex, $n=11$ for control and $n=11$ for HD; Mann-Whitney U-test, ${ }^{*} P<0.05,{ }^{* *} P<0.01$ ). (c) Immunohisto chemistry with RD4 and HT-7 antibodies in striatum (St) and cortex (Cx) of HD brain. Arrows indicate neurons with TNRs. Scale bars, $7.5 \mu \mathrm{m}$ (for St, top) and $10 \mu \mathrm{m}$ (for $\mathrm{Cx}$, bottom). (d) RD4 immunohistochemistry in hippocampus (Hipp) of AD brain. Arrows indicate neurons with TNRs. PT, pretangle neuron. Scale bar, $10 \mu \mathrm{m}$. (e) Immunohistochemistry with T22 antibody in cortex of HD brain. The arrow indicates a neuron with a TNR. Scale bar, $5 \mu \mathrm{m}$. (f) HT-7 immunofluorescence (green) and DAPI nuclear counterstaining (blue) in an HD cortical neuron. White arrows indicate the endings of the nuclear groove that harbors the tau-positive rod. Scale bar, $2.5 \mu \mathrm{m}$. (g) Immunoelectron microscopy analysis of HD neurons with HT-7-positive rods. Red arrows indicate the diaminobenzidine precipitate. The ordered filamentous ultrastructure of the immunolabeled material filling the nuclear indentations is shown. Scale bars, $2 \mu \mathrm{m}$.

(h) Immunohistochemistry with SRSF6 antibody in HD striatum (left; arrow indicates a neuron with SRSF6-positive inclusion; scale bar, $10 \mu \mathrm{m})$ and double immunofluorescence with antibodies to SRSF6 (green) and Htt (EM48, red) (right; scale bar, $2.5 \mu \mathrm{m}$ ) showing SRSF6 accumulation in an Htt IB. (i) Western blot analysis of SRSF6 and p-SR proteins (with $1 \mathrm{H} 4$ antibody for p-SR) in total homogenates from striatum of control and HD brains. Histogram shows quantification of protein abundance (SRSF6 and the 55-kDa p-SR) with respect to control ( $n=8$; Student's $t$-test; $\left.{ }^{* *} P<0.01,{ }^{* * *} P<0.001\right)$. (j) Quantitative RT-PCR analysis of 3R and 4R tau mRNA isoforms in SH-SY5Y cells transfected with GFP or with GFP fused to Htt with normal (Q17) or expanded (Q72) exon 1 ( $n=8$; Student's $t$-test; $\left.{ }^{*} P<0.05\right)$. Data are expressed as mean \pm s.e.m.

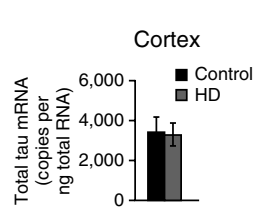

b
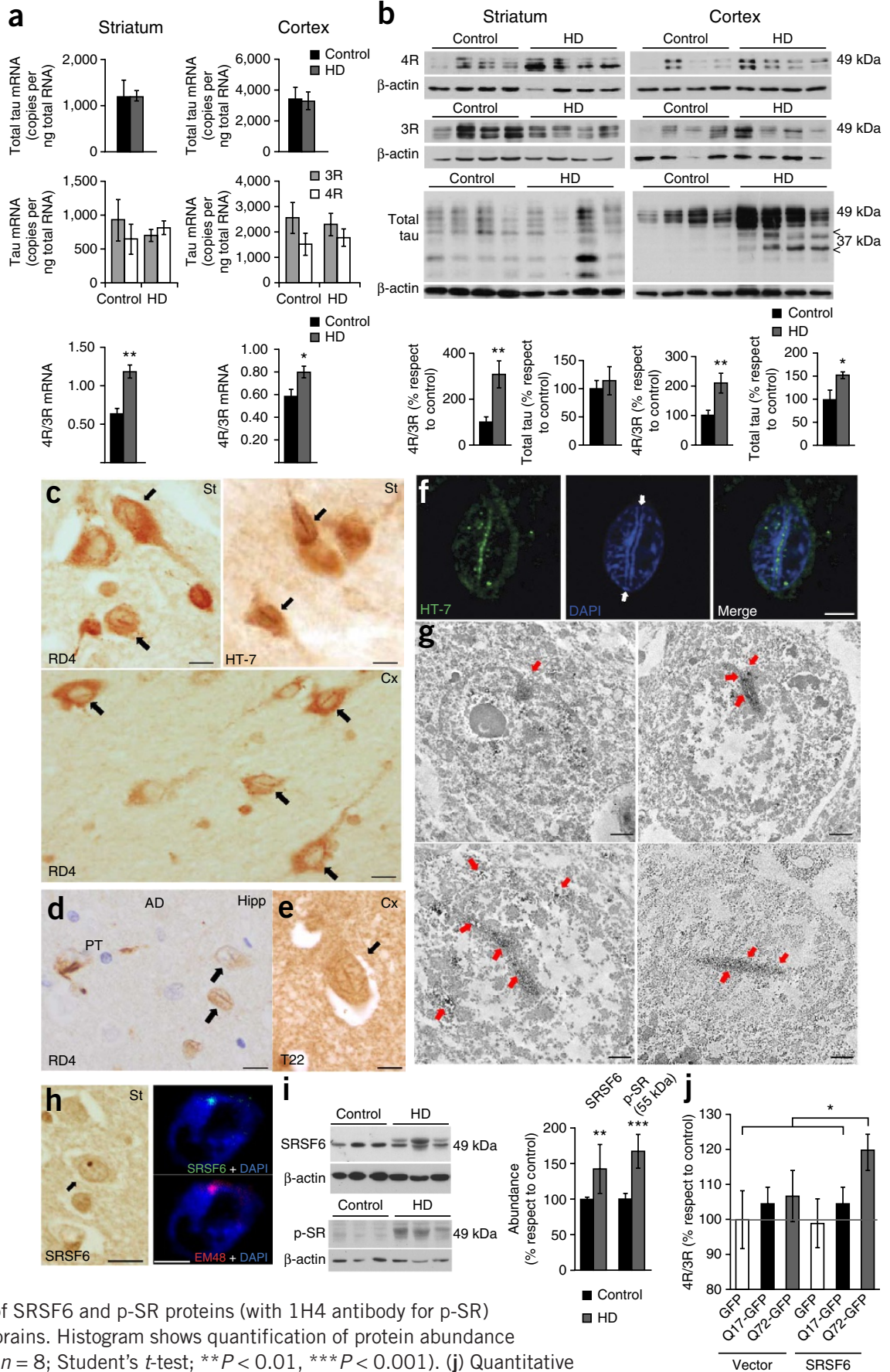

We then performed immunohistochemistry with the 4R-specific antibody (RD4) and with antibodies that recognize total tau (Tau5 and HT-7). Notably, the three antibodies detected rod-like tau deposits that partially or totally spanned the neuronal nuclear space (Fig. 1c and Supplementary Fig. 3). These neuronal tau nuclear rods (TNRs) were detected both in striatum and in cortex of each analyzed HD brain $(n=24)$, with abundance ranging from moderate to very high (Supplementary Table 1). On the contrary, in striatum of the control brains $(n=23)$, TNRs were virtually absent: they were detected in only three or fewer cases, depending on the antibody, and they were always scarce. The incidence of TNRs in cortex of the control brains was similar, although the abundance was slightly higher. Apart from TNRs, the three antibodies detected granular cytoplasmic tau deposits, which often form perinuclear rings in cortical and striatal neurons, in subjects with HD (Fig. 1c and Supplementary Fig. 3); these deposits were much less abundant in control subjects. We then wondered whether the TNRs found in brains of subjects with HD could also be found in those of individuals with a classic tauopathy. For this, we stained hippocampus of ten brains of subjects with $\mathrm{AD}$ with the RD4, Tau 5 and HT-7 antibodies, and we found that TNRs are also present in $\mathrm{AD}$ hippocampus and seemed to be more abundant at early Braak stages with low or mild neurofibrillary tangle pathology (Fig. 1d). 
As tau lesions in classic tauopathies such as progressive supranuclear palsy or Pick's disease, regardless of a shift in tau isoforms toward predominantly $4 \mathrm{R}$ or predominantly $3 \mathrm{R}$, are not exclusively composed of $3 \mathrm{R}$ or $4 \mathrm{R}$ isoforms, we reasoned that TNRs in striatum and cortex of patients with HD could also contain $3 \mathrm{R}$. We confirmed this by immunohistochemistry with the RD3 antibody (Supplementary Fig. 3 and Supplementary Table 1). Similarly, the comparison of the Sarkosyl-soluble and Sarkosyl-insoluble tau fractions in cortex of control subjects and patients with HD, apart from revealing a tendency to a higher ratio of insoluble to soluble total tau in HD than in control samples (Supplementary Fig. 4a), also confirmed the presence of both isoforms in the Sarkosyl-insoluble fraction (Supplementary Fig. $\mathbf{4 b}$ and data not shown). However, the Sarkosyl-insoluble tau in HD brains was much less abundant than was typically observed in AD samples (Supplementary Fig. 4a), and it did not contain phosphorylated tau (Supplementary Fig. 4c) - as expected, as tau is not hyperphosphorylated in cortex of HD brains (Supplementary Fig. 4d). In good agreement with these results, we did not detect TNRs by immunohistochemistry with antibodies that recognize phosphorylated epitopes of tau such as AT- 8 or PHF-1. However, we did detect TNRs in HD brains via immunohistochemistry with the T22 antibody, which recognizes oligomeric tau (Fig. 1e).

The shape and localization of TNRs suggested that they might coincide with the neuronal nuclear indentations detected by electron microscopy in the early 1980s, before the identification of the mutation underlying HD, in frontal cortex and striatum of subjects with HD but not in nonchoreic control subjects ${ }^{9,10}$; these indentations are also seen in patients with dentatorubropallidoluysian atrophy ${ }^{11}$ and in cell and mouse models of polyglutamine disease ${ }^{12-14}$. To confirm that TNRs span the neuronal nuclear space, we performed confocal analysis of brain sections from individuals with HD who were subjected to HT-7 immunofluorescence and DAPI nuclear counterstaining. DAPI staining revealed the presence of nuclear grooves that are filled by HT-7-positive TNRs (Fig. 1f). Immunoelectron microscopy analysis further confirmed that TNRs occupy neuronal nuclear indentations and uncovered an ordered filamentous ultrastructure of the immunolabeled material (Fig. 1g).

Tau and huntingtin (Htt) do not interact, as evidenced by the fact that they do not coimmunoprecipitate (Supplementary Fig. 5) and that they are restricted to their respective deposits, tau in the TNRs and Htt in the spheroid inclusion bodies (IBs). However, both histopathological hallmarks coincide in a subset of neurons. Double immunofluorescence with antibodies to tau (HT-7) and to ubiquitin, a covalently linked protein tag that is enriched in IBs, revealed that in cortex of an HD brain, $25.6 \%$ of the TNR-containing neurons also showed ubiquitin-positive nuclear IBs and $21.4 \%$ of the neurons with ubiquitin-positive nuclear IBs also showed TNRs (Supplementary Fig. 5).

Regarding the mechanism linking HTT mutation to tau alteration, Bates and colleagues ${ }^{8}$, using bioinformatic methods, recently predicted that the serine/arginine-rich splicing factor-6 (SRSF6, also known as SRp55) binds CAG repeats according to the 6-mer YRCRKM (T/C-A/G-C-A/G-G/T-A/C) consensus. They also showed, by molecular biology in vitro approaches, that SRSF6 binds the CAG repeat in mutant Htt mRNA. More recently, Krainer and colleagues ${ }^{15}$ reported an extended 9-mer consensus sequence for SRSF6 binding, out of which the last eight nucleotides provide a perfect match with CAGCAGCA. Notably, SRSF6 is a well-known modulator of tau exon 10 alternative splicing ${ }^{16}$. Here we show that SRSF6 is altered in brains of subjects with HD, as it accumulates into Htt IBs (Fig. 1h), its total abundance is increased, and a higher-molecular-weight band appears that is consistent with an increase in phosphorylated SRSF6 (Fig. 1i). We confirmed this by western blot analysis with the $1 \mathrm{H} 4$ antibody, which recognizes phosphorylated SR (p-SR) proteins (Fig. 1i). Recently, SRSF6 has been shown to favor production of 4R tau in SH-SY5Y human neuroblastoma cells ${ }^{16}$. We thus transfected SH-SY5Y cells with versions of $\mathrm{N}$-terminal Htt with normal or expanded polyQ repeats fused to GFP, and we observed a tendency toward an increased $4 \mathrm{R} / 3 \mathrm{R}$ tau mRNA ratio in cells transfected with expanded-polyQ Htt (Fig. 1j). This effect was magnified and reached statistical significance upon cotransfection of SRSF6 (Fig. 1j).

To explore whether an increased $4 \mathrm{R} / 3 \mathrm{R}$ tau ratio and increased total tau levels confer a gain of toxicity in HD pathogenesis, we decided to cross R6/1 HD transgenic mice, which develop a rapidly progressing HD-like phenotype, into heterozygous $\left(\mathrm{Mapt}^{+/-}\right)$and homozygous $\left(\mathrm{Mapt}^{-/-}\right)$tau knockout backgrounds. We first confirmed that R6/1 mice show SRSF6 alterations similar to those seen in subjects with HD, such as accumulation in Htt IBs and increased phosphorylation (Fig. 2a,b). We then verified that despite the fact that rodents express mainly $4 \mathrm{R}$ mRNA isoforms ${ }^{17,18}$, $\mathrm{R} 6 / 1$ mice still show a tendency toward an increased $4 \mathrm{R} / 3 \mathrm{R}$ ratio at both the mRNA and protein levels in striatum and cortex (Fig. 2 c,d). In addition, R6/1 mice show significantly increased $4 \mathrm{R}$ levels in striatum compared to wild-type (nontransgenic) mice (1.41-fold increase, $P=0.04$ ), and they also show significantly increased total tau levels in striatum and cortex compared to wild-type mice (1.42-fold increase, $P=0.04$ and 1.49-fold increase, $P=0.02$; respectively, Fig. 2d). We obtained similar results in HD94 mice, which have a milder, slowly progressing phenotype and a much longer lifespan than R6/1 mice. More precisely, we observed a similar increase in total tau levels in cortex of 20-month-old HD94 mice, along with a significant increase in the $4 \mathrm{R} / 3 \mathrm{R}$ tau ratio both in striatum and in cortex (Supplementary Fig. 6). We also verified that, although less abundant than in human HD brains, TNRs are also present in the brains of R6/1 and HD94 mice (Fig. 2e,f). Next, upon breeding $\mathrm{Mapt}^{+/-}$mice with R6/1 Mapt ${ }^{+/-}$ mice, we obtained R6/1Mapt $t^{+/+}, \mathrm{R} 6 / 1 \mathrm{Mapt}^{+/-}$and R6/1Mapt ${ }^{-/-}$mice according to the expected Mendelian distribution. We monitored these mice for evolution of body weight and for behavioral characterization in activity cages (to appraise global motor activity) and in the accelerating rotarod apparatus (to assess motor coordination). As expected, we first verified that R6/1 $\mathrm{Mapt}^{+/-}$and R6/1 $\mathrm{Mapt}^{-/-}$ mice showed partial and absolute reduction of tau levels, respectively (Fig. 2g). Histopathological analysis also confirmed that TNRs can also be detected in R6/1 Mapt ${ }^{+/-}$mice but not in R6/1 $\mathrm{Mapt}^{-/-}$mice (Supplementary Fig. 7) and that Mapt gene dosage did not affect the number of striatal Htt IBs, although these were slightly smaller sized in R6/1 Mapt ${ }^{+/-}$and R6/1 Mapt $t^{-/-}$mice. SRSF6 accumulation in IBs and the number of cleaved caspase-3-positive cells in striatum were not affected by the tau knockout mutation either. Regarding functional readouts of $\mathrm{R} 6 / 1$ mice, the progressive body weight loss experienced by HD transgenic mice was similar in R6/1 Mapt $t^{+/+}, \mathrm{R} 6 / 1 \mathrm{Mapt}^{+/-}$ and R6/1 Mapt ${ }^{-/-}$mice (Fig. 2h). However, R6/1 Mapt ${ }^{+/-}$and R6/1 $\mathrm{Mapt}^{-/-}$mice performed better than R6/1 Mapt $t^{+/+}$mice in the rotarod test at early symptomatic (12 weeks) and fully symptomatic (16 weeks) ages (Fig. 2i). Similarly, the significant hypoactivity observed in R6/1 $\mathrm{Mapt}^{+/+}$mice at late symptomatic ages, as evidenced by a decrease in vertical counts and in ambulatory distance in the activity cage, was attenuated in R6/1 Mapt ${ }^{+/-}$and R6/1 Mapt $t^{-/-}$mice (Fig. 2j). Together, the rotarod and activity cage results demonstrate that tau contributes to the motor phenotype of HTT transgenic mice. 
a

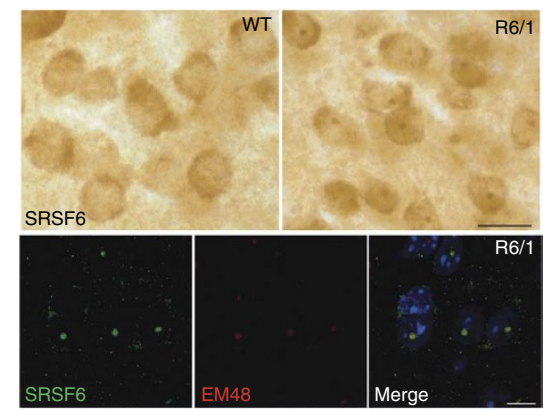

b

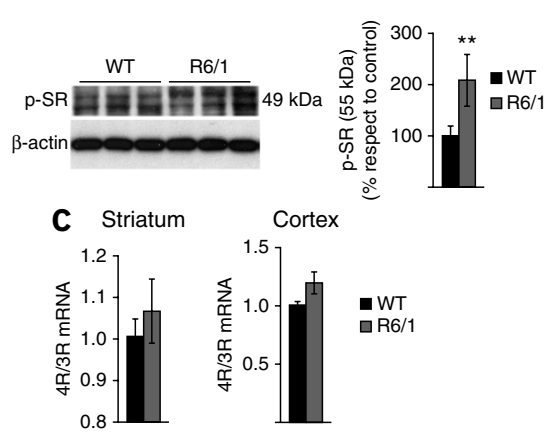

d

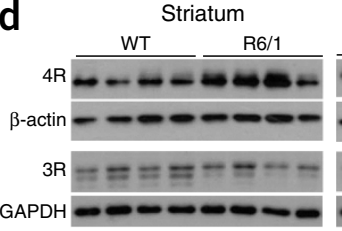

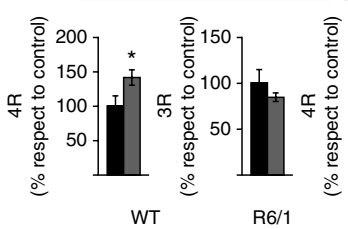

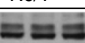
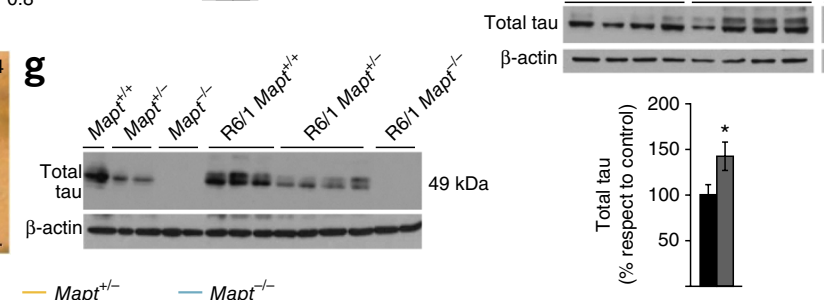
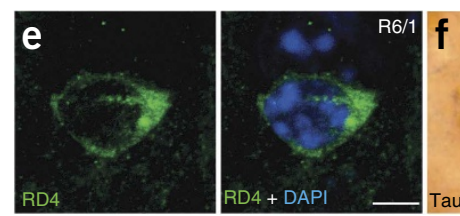

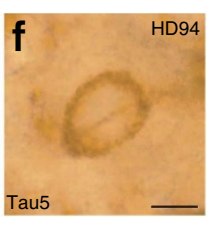

$-\mathrm{Mapt}^{+/+}$

- R6/1 Mapt

h

phosphorylation) in mutar transgenic mice, increased $4 \mathrm{R}$ tau and total tau protein levels, presence of TNRs and attenuation of motor deficit by genetic reduction of tau. (a) Immunohistochemistry with SRSF6 antibody in striatum of 14-week-old wild-type (WT) and R6/1 mice (top; scale bar, $20 \mu \mathrm{m}$ ) and double immunofluorescence with antibodies to SRSF6 (green) and Htt (EM48, red) (bottom; scale bar, $10 \mu \mathrm{m}$ ) showing SRSF6 accumulation in Htt IBs. (b) Western blot analysis of p-SR using $1 \mathrm{H} 4$ antibody in striatum of 14 -week-old wild-type and R6/1 mice ( $n=6$; Student's $t$-test; ${ }^{* *} P<0.01$ ). (c) Quantitative RT-PCR analysis of $4 \mathrm{R} / 3 \mathrm{R}$ tau mRNA isoform ratio in striatum and cortex of 14-week-old R6/1 mice ( $n=5$ for WT and $n=7$ for R6/1). (d) Western blot analysis of $3 \mathrm{R}$ and $4 \mathrm{R}$ tau isoforms with the RD3- and RD4-specific antibodies and of total tau with the 7.51 antibody in striatum and cortex of wild-type and R6/1 mice (the position of the 49-kDa molecular weight marker is indicated; $n=7$; Student's $t$-test; * $P<0.05$ ). (e) RD4 immunofluorescence (green) and DAPI nuclear counterstaining (blue) showing a TNR in a R6/1 neuron. Scale bar, $5 \mu \mathrm{m}$. (f) Detection of a TNR by immunohistochemistry with Tau 5 antibody in an HD94 neuron. Scale bar, $5 \mu \mathrm{m}$. (g) Western blot analysis of total tau (7.51 antibody) in striatum of wild-type $\left(\right.$ Mapt $\left.^{+/+}, n=1\right), \mathrm{Mapt}^{+/-}(n=2)$, $\mathrm{Mapt}^{-/-}(n=2), \mathrm{R} 6 / 1 \mathrm{Mapt}^{+/+}(n=3), \mathrm{R6} / 1 \mathrm{Mapt}^{+/-}(n=4)$, and R6/1 Mapt ${ }^{-/-}$mice $(n=2)$. (h) Evolution of body weight of $\mathrm{Mapt}^{+/+}(n=9), \mathrm{Mapt}^{+/-}$ $(n=19), \mathrm{Mapt}^{-/-}(n=5), \mathrm{R} 6 / 1 \mathrm{Mapt}^{+/+}(n=12), \mathrm{R} 6 / 1 \mathrm{Mapt}^{+/-}(n=17)$ and R6/1 Mapt ${ }^{-/-}(n=9)$ mice. (i) Evolution of motor coordination in the accelerating rotarod test of $\mathrm{Mapt}^{+/+}(n=5), \mathrm{Mapt}^{+/-}(n=9), \mathrm{Mapt}^{-/-}(n=5), \mathrm{R} 6 / 1 \mathrm{Mapt}^{+/+}(n=6), \mathrm{R} 6 / 1 \mathrm{Mapt}^{+/-}(n=14)$ and R6/1 Mapt ${ }^{-/-}(n=8)$ mice at 12 and 16 weeks of age. Analysis of variance (ANOVA), followed by minimum significant difference or Games-Howell, ** $P<0.01,{ }^{*} P<0.05$. (j) Open-field test of $\mathrm{Mapt}^{+/+}(n=9), \mathrm{Mapt}^{+/-}(n=11), \mathrm{Mapt}^{-/-}(n=3), \mathrm{R6} / 1 \mathrm{Mapt}^{+/+}(n=8), \mathrm{R} 6 / 1 \mathrm{Mapt}^{+/-}(n=13)$ and R6/1 Mapt ${ }^{-/-}(n=4) \mathrm{mice}$ at 25 weeks of age. For ambulatory distance, ANOVA, followed by minimum significant difference, ${ }^{*} P<0.05$; for vertical counts, Kruskall-Wallis, followed by Bonferroni post hoc test, ${ }^{*} P<0.008$. Data are expressed as mean \pm s.e.m.
In summary, here we show that the HTT mutation leads to pathogenic tau alterations, namely an increase of the $4 \mathrm{R} / 3 \mathrm{R}$ tau isoform ratio and an increase in total tau content. In addition, we found nuclearspanning rod-like tau deposits (TNRs) in HD brains, which we also found in AD brains. Finally, the attenuated motor phenotype of HTT transgenic mice with genetic tau reduction demonstrates a role of tau in HD pathogenesis. However, it should be noted that additional work needs to be done to elucidate whether the three key findings in this study-altered $4 \mathrm{R} / 3 \mathrm{R}$ tau splicing resulting in an imbalance $4 \mathrm{R}$ and $3 \mathrm{R}$ isoforms, presence of TNRs and in vivo effects of reduced tau expression in mutant HTT transgenic mice-are functionally linked to each other. For instance, if TNRs were found in brains of individuals with FTPD-17 with intronic mutations, this would strongly suggest that an imbalance of $4 R$ and $3 R$ tau isoforms is sufficient to cause TNR formation. At this time, we do not know whether TNRs contribute to $\mathrm{HD}$ pathogenesis. One possible scenario is that an increased $4 \mathrm{R} / 3 \mathrm{R}$ tau isoform ratio leads to altered microtubule dynamics and multiple subsequent intracellular alterations, such as a pathogenic disruption of axonal transport accompanied by a harmless bundling of microtubules from the centrosome, that induce a nuclear indentation with a high content of tau. In this case, formation of TNRs would not be pathogenic but rather a marker of altered microtubule dynamics as a consequence of an altered $4 \mathrm{R} / 3 \mathrm{R}$ tau isoform ratio. Alternatively, TNRs might compromise the integrity of the nuclear envelope and/or lead to nuclear fragmentation and neuronal death. Most of these are testable hypotheses and, as mentioned, additional experiments will need to be done to explore the interconnection and precise pathophysiological implications of the findings reported here for HD brains and models. 
Future studies will also be required to determine whether similar tau alterations can be found in the other neurological disorders caused by an expanded CAG repeat (although we could not detect TNRs in cerebellum of a very limited number of SCA1 and SCA3 brains; Supplementary Fig. 8). If tau alterations are indeed specific for HD, this might be the reason why, unlike the SCAs, HD presents with basal ganglia-associated movement alterations and cortical dementia. Finally, the incidence of TNRs needs to be thoroughly analyzed in $\mathrm{AD}$ and the rest of the classic tauopathies, particularly those with accumulation of $4 \mathrm{R}$ tau and an imbalance of $4 \mathrm{R}$ and $3 \mathrm{R}$ isoforms, such as FTDP-17. Finally, our results suggest that either gene therapy- or small molecule-based tau-related therapies currently under development for FTDP-17 and other tauopathies ${ }^{19,20}$ might also be helpful for HD.

\section{METHODS}

Methods and any associated references are available in the online version of the paper.

Note: Any Supplementary Information and Source Data files are available in the online version of the paper.

\section{ACKNOWLEDGMENTS}

We thank P. Gómez-Ramos for helpful suggestions on electron microscopy experiments and A. Rábano and E. Gelpí for advice on human sample analysis. We also thank A. Tomico, M. Lucas and the team at the CBMSO Genomics Facility for their excellent technical assistance, as well as members of the Lucas lab for helpful advice and critical reading of the manuscript. P. Davies (Albert Einstein University) provided the PHF-1 antibody to phosphorylated tau; M. Novak (Slovak Academy of Sciences) and C.M. Wischik (University of Aberdeen) provided the 7.51 antibody to total tau; R. Kayed (University of Texas) provided the T22 antibody to oligomeric tau; S. Finkbeiner (Gladstone Institute) provided Htt constructs comprising the N-terminal fragment of $\mathrm{Htt}$ with 17 or 72 CAG repeats fused to the EGFP; and A.R. Krainer (Cold Spring Harbor Laboratory) provided SRSF6 expression vector. This work was supported by Centro de Investigación Biomédica en Red de Enfermedades Neurodegenerativas (CiberNed-Instituto de Salud Carlos III) and by grants from Ministerio de Ciencia e Innovación (MICINN), Ministerio de Economía y Competitividad (MINECO), Comunidad Autónoma de Madrid, Fundación Ramón Areces and the Seventh Framework Programme of the European Commission (grant agreement 278486, project DEVELAGE). M.F.-N. was the recipient of a CSIC JAE-Pre research contract.

\section{AUTHOR CONTRIBUTIONS}

J.J.L. directed the study. M.F.-N., J.R.C., M.S.-G., J.J.M.H. and I.F. designed and performed experiments. M.F.-N., J.R.C., J.J.M.H., A.J.M.R., I.F. and J.J.L. analyzed and interpreted the data. F.H. and J.A. made intellectual contributions to experimental design and discussion. J.J.L. wrote the manuscript.

\section{COMPETING FINANCIAL INTERESTS}

The authors declare no competing financial interests.

Reprints and permissions information is available online at http://www.nature.com/ reprints/index.html.

1. Lee, V.M., Goedert, M. \& Trojanowski, J.Q. Annu. Rev. Neurosci. 24, 1121-1159 (2001).

2. Liu, F. \& Gong, C.X. Mol. Neurodegener. 3, 8 (2008).

3. Hutton, M. et al. Nature 393, 702-705 (1998).

4. Huntington's Disease Collaborative Research Group. Cell 72, 971-983 (1993).

5. Nelson, D.L., Orr, H.T. \& Warren, S.T. Neuron 77, 825-843 (2013).

6. Ranum, L.P. \& Cooper, T.A. Annu. Rev. Neurosci. 29, 259-277 (2006).

7. Mykowska, A., Sobczak, K., Wojciechowska, M., Kozlowski, P. \& Krzyzosiak, W.J. Nucleic Acids Res. 39, 8938-8951 (2011).

8. Sathasivam, K. et al. Proc. Natl. Acad. Sci. USA 110, 2366-2370 (2013).

9. Bots, G.T. \& Bruyn, G.W. Acta Neuropathol. 55, 21-22 (1981).

10. Roos, R.A. \& Bots, G.T. J. Neurol. Sci. 61, 37-47 (1983).

11. Takahashi, H. et al. Brain Res. 919, 12-19 (2001).

12. Evert, B.O. et al. Hum. Mol. Genet. 8, 1169-1176 (1999).

13. Díaz-Hernandez, M. et al. J. Neurosci. 23, 11653-11661 (2003).

14. Zander, C. et al. Hum. Mol. Genet. 10, 2569-2579 (2001).

15. Jensen, M.A., Wilkinson, J.E. \& Krainer, A.R. Nat. Struct. Mol. Biol. 21, 189-197 (2014)

16. Yin, X. et al. J. Biol. Chem. 287, 30497-30506 (2012).

17. Kosik, K.S., Orecchio, L.D., Bakalis, S. \& Neve, R.L. Neuron 2, 1389-1397 (1989).

18. Takuma, H., Arawaka, S. \& Mori, H. Brain Res. Dev. Brain Res. 142 121-127 (2003).

19. Rodriguez-Martin, T. et al. Hum. Mol. Genet. 18, 3266-3273 (2009).

20. Brunden, K.R., Trojanowski, J.Q. \& Lee, V.M. Nat. Rev. Drug Discov. 8, 783-793 (2009). 


\section{ONLINE METHODS}

Human brain tissue samples. Brain specimens used in this study from frontal cortex and striatum of subjects with $\mathrm{HD}(n=24)$ and control subjects $(n=23)$, as well as hippocampus of patients with $\mathrm{AD}(n=10)$ and cerebellum of patients with SCA1 $(n=1)$ and SCA3 $(n=3)$, were provided by the Institute of Neuropathology (HUB-ICO-IDIBELL) Brain Bank (Hospitalet de Llobregat, Spain), the Neurological Tissue Bank of the IDIBAPS Biobank (Barcelona, Spain), the Banco de Tejidos Fundación Cien (BT-CIEN, Madrid, Spain) and the Netherlands Brain Bank (Amsterdam, the Netherlands). Written informed consent for brain removal after death for diagnostic and research purposes was obtained from brain donors and/or next of kin. Procedures, information and consent forms have been approved by the Medical Ethics Committee of the VU University Medical Center (Amsterdam, the Netherlands) and the Bioethics Subcommittee of Centro Superior de Investigaciones Científicas (Madrid, Spain). The postmortem delay in tissue processing was between $3 \mathrm{~h}$ $40 \mathrm{~min}$ and $48 \mathrm{~h}$. The neuropathological examination in HD cases revealed a diagnosis of HD grade from 0 to 4 following the criteria of Vonsattel ${ }^{21}$.

Quantitative real-time RT-PCR. Total tissue RNA was extracted from striatum and cortex of subjects with HD and control subjects using the Maxwell 16 LEV simplyRNA Tissue Kit (Promega). The resulting total RNA (750 ng) was used for cDNA synthesis with a Super Script III First-Strand Synthesis SuperMix kit from Invitrogen (PN 11752250) followed by PCR with the amplification protocol $30 \mathrm{~s}$ at $95^{\circ} \mathrm{C}+\left(5 \mathrm{~s}\right.$ at $95{ }^{\circ} \mathrm{C}+5 \mathrm{~s}$ at $\left.60^{\circ} \mathrm{C}\right) \times 40+(5 \mathrm{~s}$ at 60 ${ }^{\circ} \mathrm{C}+5 \mathrm{~s}$ at $95^{\circ} \mathrm{C}$ ). Quantification was performed by real-time PCR using a CFX 384 System (Bio-Rad) in combination with SsoFast Eva Green (Bio-Rad), as per manufacturer's protocol, and $1 \mu \mathrm{L}$ of primer pair was used. Data were analyzed by GenEx 5.3.7 software (Multid AnaLyses AB). The mRNA levels were normalized first relative to total RNA and then relative to the mean of $18 \mathrm{~S}$ ribosome subunit, GADPH, $\beta$-actin and $\beta$-tubulin gene expression in each sample. The PCR primers used for human were the following: total tau forward 5'AGAGTCCAGTCGAAGATTGGGTC 3', total tau reverse 5' GGGTTTCAA TCTTTTTATTTCCTCC $3^{\prime}$; $4 \mathrm{R}$ tau forward 5' GGTGCAGATAATTAATAAG AAGCTGGA 3', 4R tau reverse 5' GTGTTTGATATTATCCTTTGAGCCAC 3'; and 3R tau forward 5' GAAGAATGTCAAGTCCAAGATCGG 3', 3R tau reverse $5^{\prime}$ GACTATTTGCACCTTCCCGC $3^{\prime}$. The PCR primers used for mouse were the following: total tau forward $5^{\prime}$ AGGGAACATCCATCACAAGC $3^{\prime}$, total tau reverse $5^{\prime}$ TGGACTCTGTCCTTGAAGTCC 3'; 4R tau forward 5' TGTCAGGTCGAAGATTGGCTC ${ }^{\prime}$, 4R tau reverse $5^{\prime}$ CTTATTAATTATCTGC ACCTTGCCAC 3'; and 3R tau forward 5' GTCAGGTCGAAGATTGGCTCTACT 3', 3R tau reverse 5' GCTTGTAGACTATTTGCACCTTGC 3'. In human samples, to determine the ratio of the $4 \mathrm{R} / 3 \mathrm{R}$ tau isoform expression in each sample by absolute quantitative PCR, the total tau, $4 \mathrm{R}$ tau and $3 \mathrm{R}$ tau amplicon obtained with the above-mentioned selective primers for total tau, $4 \mathrm{R}$ tau and 3R tau were inserted into a pMA-T plasmid that was provided by GeneART (Life Technologies). This pMA-T-Total Tau_4R Tau_3R Tau plasmid, containing one copy of each total tau, $4 \mathrm{R}$ tau and 3R tau amplicons, was digested with the AscI restriction enzyme and serially diluted to generate a calibration curve. As expected, the efficiency of the PCR reaction and the melting curve for each primer pair were the same using the standard pMA-T-Total Tau_4R Tau_3R Tau plasmid or human cDNA samples. To assess whether postmortem intervalrelated degradation differentially affects certain transcripts, samples from a subject with $\mathrm{HD}$ and a control subject were left at $4{ }^{\circ} \mathrm{C}$ for 24 and $48 \mathrm{~h}$. Next, total tissue RNA was extracted, and quantitative real-time RT-PCR was performed.

Western blotting. The different human brain regions that were stored at $-80^{\circ} \mathrm{C}$ were ground with a mortar in a frozen environment with liquid nitrogen to prevent thaw of the samples. The resulting powder was homogenized. For mice, brains were quickly dissected on an ice-cold plate. Both extracts were prepared by homogenizing the brain areas in ice-cold extraction buffer consisting of $20 \mathrm{mM}$ HEPES, $\mathrm{pH}$ 7.4, $100 \mathrm{mM} \mathrm{NaCl}, 20 \mathrm{mM} \mathrm{NaF}, 1 \%$ Triton X-100, $1 \mathrm{mM}$ sodium orthovanadate, $1 \mu \mathrm{M}$ okadaic acid, $5 \mathrm{mM}$ sodium pyrophosphate, $30 \mathrm{mM} \beta$-glycerophosphate, $5 \mathrm{mM}$ EDTA and protease inhibitors ( $2 \mathrm{mM}$ PMSF, $10 \mu \mathrm{g} / \mathrm{ml}$ aprotinin, $10 \mu \mathrm{g} / \mathrm{ml}$ leupeptin and $10 \mu \mathrm{g} / \mathrm{ml}$ pepstatin). Samples were homogenized and centrifuged at $15,000 \mathrm{~g}$ for $15 \mathrm{~min}$ at $4^{\circ} \mathrm{C}$. The resulting supernatant was collected, and protein content determined by Bradford assay. Fifteen micrograms of total protein were electrophoresed on $10 \%$ SDS-polyacrylamide gel and transferred to a nitrocellulose membrane and blocked in TBS-T ( $150 \mathrm{mM} \mathrm{NaCl}, 20 \mathrm{mM}$ Tris-HCl, pH 7.5, $0.05 \%$ Tween 20) supplemented with 5\% nonfat dry milk.

Inclusion or exclusion of exon 10 results in unique amino acid sequences in the tubulin-binding domain of either $3 \mathrm{R}$ and $4 \mathrm{R}$ tau protein isoforms. This fact has allowed the generation by de Silva et al..$^{22}$ of monoclonal antibodies that distinguish with complete specificity the $4 \mathrm{R}$ and $3 \mathrm{R}$ tau protein isoforms. These antibodies are termed RD4 (1:1,000, clone 1E1/A6, 05-804 from Millipore) and RD3 (1:1,000, clone 8E6/C11, 05-803 from Millipore), respectively. Other employed antibodies were anti-tau clone Tau5 (1:1,000, 577801 from Calbiochem), antiSRSF6 (1:1,000, LS-B5712 from LSBio), anti-p-SR clone 1H4 (1:1,000, MABE50 from Millipore), anti-tau clone 5E2 (1:1,000, 05-348 from Millipore), anti-PHF1 (1:1,000, provided by P. Davies), anti-tau clone 7.51 (1:1,000, provided by M. Novak and C.M. Wischik), anti-tau clone AT8 (1:1,000, MN1020 from Thermo Scientific), anti-GADPH (1:2,000, ab9483 from Abcam) and $\beta$-actin clone AC-74 (1:4,000, A5316 from Sigma-Aldrich).

The membranes were incubated with the primary antibody overnight at $4{ }^{\circ} \mathrm{C}$ in TBST supplemented with $5 \%$ nonfat dried milk, washed in TBS-T and then incubated with secondary HRP-conjugated anti-mouse IgG (P0447 from DAKO Cytomation) and developed using the ECL detection kit (Perkin Elmer).

Immunohistochemistry and immunofluorescence. For human samples, formalin-fixed $(4 \%, 24 \mathrm{~h})$, paraffin-embedded tissue from cortex and striatum were used. Sections $(5-\mu \mathrm{m}$ thick) were mounted on superfrost-plus tissue slides (Menzel-Gläser) and deparaffinized.

Mice were killed using $\mathrm{CO}_{2}$ and brains immediately removed and dissected on an ice-cold plate. Left hemispheres were processed for histology, placed in $4 \%$ paraformaldehyde in Sorensen's phosphate buffer (PFA) overnight and then immersed in $30 \%$ sucrose in PBS for $72 \mathrm{~h}$. Once cryoprotected, the samples were included in OCT compound (Sakura Finetek Europe), frozen and stored at $-80^{\circ} \mathrm{C}$ until use. 30- $\mu \mathrm{m}$ sagittal sections were cut on a CM $1950 \mathrm{Ag}$ Protect freezing microtome (Leica) and collected and stored free-floating in glycol containing buffer ( $30 \%$ glycerol, $30 \%$ ethylene glycol in $0.02 \mathrm{M}$ phosphate buffer) at $-20^{\circ} \mathrm{C}$. Subsequently, sections were immersed in $0.3 \% \mathrm{H}_{2} \mathrm{O}_{2}$ in methanol for $30 \mathrm{~min}$ to quench endogenous peroxidase activity and treated in $10 \mathrm{mM} \mathrm{pH} 6.0$ citrate buffer heated by microwave for $15 \mathrm{~min}$ for antigen retrieval. For immunohistochemical staining, slides were blocked for $1 \mathrm{~h}$ in PBS containing 0.5\% FBS, $0.3 \%$ Triton X-100 and 1\% BSA (Sigma-Aldrich). Sections were incubated overnight at $4{ }^{\circ} \mathrm{C}$ in PBS containing $0.3 \%$ Triton X-100 and $1 \%$ BSA with the corresponding primary antibodies: RD4 clone 1E1/A6 (1:80, 05-804 from Millipore), anti-tau clone HT-7 (1:200, MN1000 from Thermo Scientific), anti-tau clone Tau5 (1:200, 577801 from Calbiochem), RD3 clone 8E6/C11 (1:4,000, 05-803 from Millipore), T22 recognizing oligomeric $\operatorname{tau}^{23}$ (1:100, provided by R. Kayed), anti-SRSF6 (1:400, LS-B5712 from LSBio), anti-huntingtin clone EM48 (1:100, MAB5374 from Millipore) and anti-cleaved caspase-3 (1:50, 9661 from Cell Signaling). Finally, brain sections were incubated in avidin-biotin complex using the Elite Vectastain kit (Vector Laboratories). Chromogen reactions were performed with diaminobenzidine (SIGMAFAST DAB, Sigma) for $10 \mathrm{~min}$. Sections were mounted on glass slides and coverslipped with Mowiol (Calbiochem). Images were captured using an Olympus BX41 microscope with an Olympus camera DP-70 (Olympus Denmark A/S). For immunofluorescence, after antigen retrival, slides were pretreated with $0.1 \%$ Triton X-100 for $15 \mathrm{~min}, 1 \mathrm{M}$ glycine for $30 \mathrm{~min}$ and blocking solution (1\% BSA and $0.1 \%$ Triton X-100) for $1 \mathrm{~h}$. Sections were then incubated overnight at $4{ }^{\circ} \mathrm{C}$ with the following primary antibodies in blocking solution: anti-tau clone HT-7 (1:50, MN1000 from Thermo Scientific), anti-ubiquitin (1:100, Z0458 from Dako), anti-SRSF6 (1:200, LS-B5712 from LSBio) and anti-huntingtin clone EM48 (1:1,000, MAB5374 from Millipore). The following day, sections were washed in PBS. Next, sections were incubated in avidin-biotin complex using the Elite Vectastain kit (Vector Laboratories) for $1 \mathrm{~h}$, and, after that, sections were incubated with Streptavidin-Alexa 488 (1:500, Invitrogen) and donkey anti-rabbit Alexa 555 (1:500, Invitrogen) secondary antibodies for $1 \mathrm{~h}$. Nuclei were counterstained with DAPI $(1: 4,000$, Calbiochem). Finally, sections were incubated with Sudan Black for $10 \mathrm{~min}$ and mounted on glass slides, coverslipped with DePeX (Serva) and maintained at $4{ }^{\circ} \mathrm{C}$. Images were acquired with a laser confocal LSM710 system coupled to the invert Axioobserver microscope with a $63 \times, 1.4$ numerical aperture 
oil-immersion objective using the Zen2010B sp1 software (Carl Zeiss). Sequential optic sections $(-0.8 \mu \mathrm{m})$ were acquired in $z$ stacks. Images were processed using Image $1.45 \mathrm{~s}$.

Electron microscopy. For electron microscopy, immunostained sections with HT-7 (MN1000 from Thermo Scientific) from human cortex were processed. Briefly, sections were postfixed in $2 \% \mathrm{OsO}_{4}$ for $1 \mathrm{~h}$, dehydrated, embedded in Araldite and flat-mounted in Formvar-coated slides using plastic cover slips. After polymerization, selected areas were photographed, trimmed, reembedded in Araldite and resectioned at $1 \mu \mathrm{m}$. These semi-thin sections were rephotographed and resectioned in ultrathin sections. The ultrathin sections were observed in a Jen 2010 Joel transmission electron microscope (Peabody), without heavy metal staining to avoid artifactual precipitates.

Cell culture. SH-SY5Y neuroblastoma cell line was grown on DMEM, 10\% FBS and antibiotics. Cells were transfected using Lipofectamine 2000 (Life Technologies) according to manufacturer's instructions. Htt constructs comprised pEGFP-1 with the N-terminal fragment of $\mathrm{Htt}$ with 17 or $72 \mathrm{CAG}$ repeats fused to the EGFP (provided by S. Finkbeiner). SRSF6 expression vector was provided by A.R. Krainer.

Examination of Sarkosyl-insoluble fractions. Fresh samples from the frontal cortex of subjects with Alzheimer's disease (AD, used as a positive control for 3R-4R tauopathy) or HD and of control subjects (about $1 \mathrm{~g}$ ) were homogenized in a glass tissue grinder in $10 \mathrm{vol}(\mathrm{w} / \mathrm{v})$ of cold suspension buffer consisting of $10 \mathrm{mM}$ Tris- $\mathrm{HCl}$ (pH 7.4), $0.8 \mathrm{M} \mathrm{NaCl}, 1 \mathrm{mM}$ EGTA, $10 \%$ sucrose, $0.1 \mathrm{mM}$ phenylmethylsulfonyl fluoride, $2 \mu \mathrm{g} / \mathrm{ml}$ aprotinin, $10 \mu \mathrm{g} / \mathrm{ml}$ leupeptin and $5 \mu \mathrm{g} / \mathrm{ml}$ pepstatin. The homogenates were first centrifuged at $20,000 \mathrm{~g}$, and the supernatant (S1) was retained. The pellet (P1) was rehomogenized in 5 units of homogenization buffer and recentrifuged. The two supernatants $(\mathrm{S} 1+\mathrm{S} 2)$ were then mixed and incubated with $N$-lauroylsarcosinate (Sarkosyl) $10 \%$ for $1 \mathrm{~h}$ at room temperature while shaking. Samples were then centrifuged for $1 \mathrm{~h}$ at $100,000 \mathrm{~g}$ in a Ti 70 Beckman rotor. Sarkosyl-insoluble pellets (P3) were resuspended $(0.2 \mathrm{ml} / \mathrm{g}$ of starting material) in $50 \mathrm{mM}$ Tris- $\mathrm{HCl}$ (pH 7.4). Protein concentrations were determined by the BCA method and $10 \%$ sodium dodecyl sulfate-polyacrylamide gel electrophoresis (SDS-PAGE) was run using a MiniProtean system (Bio-Rad). $50 \mu \mathrm{g}$ of protein was loaded in each lane with loading buffer containing $0.125 \mathrm{M}$ Tris (pH 6.8), $20 \%$ glycerol, $10 \%$ mercaptoethanol, $4 \%$ SDS and $0.002 \%$ bromophenol blue. Samples were heated at $95{ }^{\circ} \mathrm{C}$ for $5 \mathrm{~min}$ before gel loading. The proteins were then transferred to nitrocellulose membranes (Amersham) using an electrophoretic chamber system (Trans-Blot Electrophoretic Transfer Cell, Bio-Rad). Nonspecific binding sites were blocked with Tris-buffered saline solution, $\mathrm{pH} 7.4$, with $0.1 \%$ Tween- 20 (TBST) containing $5 \%$ skim milk for $30 \mathrm{~min}$ and incubated with one of the primary antibodies for $1 \mathrm{~h}$ at room temperature. The experiments were performed using the following primary antibodies: anti-tau clone Tau 5 (1:1,000, 577801 from Calbiochem), RD3 clone 8E6/C11 (1:1,000, 05-803 from Millipore) and anti-P-Tau Ser422 (1:1,000, AB9664 from Calbiochem). After washing, membranes were incubated with the secondary antibody labeled with horseradish peroxidase (Dako), diluted 1:1,000 for $1 \mathrm{~h}$ at room temperature, washed again and developed with the chemiluminescence ECL Western Blotting system (Amersham). Membranes were then exposed to autoradiographic films (Hyperfilm ECL, Amersham).

Coimmunoprecipitation. Extract from human striatum (control or HD) was precleared and then incubated with Extactacruz matrix B using huntingtin antibody N-18 (1:100, 8767 from Santa Cruz Biotechnology). Matrix was washed three times using lysis buffer and boiled; western blotting was performed.

Animals. R6/1 mice transgenic for the human exon 1 mutant HTT gene in the B6CBAF1 background ${ }^{24}$ and tau-knockout $\left(\mathrm{Mapt}^{-{ }^{--}}\right)$mice in the B6 background ${ }^{25}$ were used. To obtain R6/1 mice in $\mathrm{Mapt}^{+/-}$and $\mathrm{Mapt}^{-/-}$backgrounds, male R6/1 mice were crossed with $\mathrm{Mapt}^{+/-}$females. From the offspring, a second cross was done with $\mathrm{Mapt}^{+/-}$male mice and R6/1 $\mathrm{Mapt}^{+/-}$females to achieve the six different genotypes: wild-type, $\mathrm{Mapt}^{+/-}, \mathrm{Mapt}^{-/-}, \mathrm{R} 6 / 1 \mathrm{Mapt}^{+/+}, \mathrm{R} 6 / 1 \mathrm{Mapt}^{+/-}$ and R6/1 $\mathrm{Mapt}^{-1-}$. HD94 mice were generated as previously described ${ }^{26}$. All mice were housed at the Centro de Biología Molecular "Severo Ochoa" animal facility. Mice were housed four per cage with food and water available ad libitum and maintained in a temperature-controlled environment on a 12-h light-dark cycle with light onset at 7:00 a.m. For behavioral analysis, mice were tested at the ages of 12 and 16 weeks. For behavioral studies, males and females were used interchangeably. Animal housing and maintenance protocols followed the guidelines of Council of Europe Convention ETS123, recently revised as indicated in the Directive 86/609/EEC. Animal experiments were performed under protocols (P15/P16/P18/P22) approved by the Centro de Biología Molecular Severo Ochoa Institutional Animal Care and Utilization Committee (Comité de Ética de Experimentación Animal del CBM, CEEA-CBM), Madrid, Spain.

Behavioral testing. The rotarod test was performed with accelerating rotarod apparatus (Ugo Basile). Mice were pretrained for $2 \mathrm{~d}$ at a constant speed, 4 r.p.m. the first day over $1 \mathrm{~min}$ four times or 8 r.p.m. over $1 \mathrm{~min}$ four times the second day. On the third day, the rotarod was set to accelerate from 4 to 40 r.p.m. over $5 \mathrm{~min}$, and mice were tested four times. During accelerating trials, the latency to fall from the rod was measured. The number of animals used for this test was as follows: wild-type, $n=5$; $\mathrm{Mapt}^{+/-}, n=9 ; \mathrm{Mapt}^{-/-}, n=5 ; \mathrm{R} 6 / 1 \mathrm{Mapt}^{+/+}, n=6$; R6/1 Mapt $t^{+/-}, n=14$; and R6/1 Mapt ${ }^{-/-}, n=8$.

For the open field test, locomotor activity was measured in clear Plexiglas boxes measuring $43.2 \mathrm{~cm} \times 43.2 \mathrm{~cm}$, outfitted with photobeam detectors for monitoring horizontal and vertical activity. Activity levels were recorded with an MED Associates' Activity Monitor (MED Associates, St. Albans, VT). Locomotor activity data were collected via a PC and analyzed with the MED Associates' Activity Monitor Data Analysis software. Mice were placed in a corner of the open-field apparatus and left to move freely. Variables recorded included vertical counts and ambulatory distance $(\mathrm{cm})$. Data were individually recorded for each animal over $15 \mathrm{~min}$. The number of animals used for this test was as follows:: wild-type, $n=9 ; \mathrm{Mapt}^{+/-}, n=11 ; \mathrm{Mapt}^{-/-}, n=3$; R6/1 $\mathrm{Mapt}^{+/+}$, $n=8 ; \mathrm{R} 6 / 1 \mathrm{Mapt}^{+/-}, n=13$; and R6/1 $\mathrm{Mapt}^{-/-}, n=4$. As no sex differences were found among genotypes, males and females were used interchangeably. Mice were weighed every 2 weeks from the age of 14 weeks.

Analysis of number and size of Htt inclusion bodies in mouse brain. Four regularly spaced sections of each mouse brain were immunostained for antiN-terminal Htt (1:100, MAB5374, Millipore). Digital images of striatal Htt inclusions were captured with an Olympus Bx 51 microscope, Color View IIIu digital camera with a $40 \times$ objective lens with the help of the Olympus Soft Imaging System. The number and size of inclusions were determined using the ImageJ software with the Analyze Particles routine (size 2.89-14.45 $\mu \mathrm{m}^{2}$, threshold 0-75, circularity $0.81-1.00$ ). Data are represented as the mean \pm s.e.m.

Statistical analyses. Statistical analysis was performed with SPSS 19.0 (SPSS Statistic IBM). Data are represented as means \pm s.e.m. The normality of the data was analyzed by Shapiro-Wilk test. For two-group comparison, two-tailed Student's $t$-test (data with normal distribution) or Mann-Whitney $U$-test (data with non-normal distribution) was performed. For multiple comparisons, data with a normal distribution were analyzed by one-way ANOVA test followed by a minimum significant difference (MSD) or a Games-Howell post hoc test. Statistical significance of nonparametric data was determined by Kruskal-Wallis test when analyzing all experimental groups followed by a Mann-Whitney $U$-test for analysis of paired genotypes, and Bonferroni correction was applied. A critical value for significance of $P<0.05$ was used throughout the study. No statistical method was used to predetermine sample size. The investigators were blinded to genotype during experiments in the immunohistochemical counting of the TNRs and behavioral tests. The experiments were not randomized.

21. Vonsattel, J.P. et al. J. Neuropathol. Exp. Neurol. 44, 559-577 (1985).

22. de Silva, R. et al. Neuropathol. Appl. Neurobiol. 29, 288-302 (2003).

23. Lasagna-Reeves, C.A. et al. FASEB J. 26, 1946-1959 (2012).

24. Mangiarini, L. et al. Cell 87, 493-506 (1996).

25. Dawson, H.N. et al. J. Cell Sci. 114, 1179-1187 (2001).

26. Yamamoto, A., Lucas, J.J. \& Hen, R. Cell 101, 57-66 (2000). 